\title{
Effect of vitiligo treatment by compound Glycyrrhizin combined with fractional laser and Triamcinolone Acetonide injection on T Lymphocyte subpopulation
}

\author{
Ling $\mathrm{Li}^{1}$, Lei Gao ${ }^{2}$, Yifan $\mathrm{Zhao}^{3}$
}

\begin{abstract}
Objectives: To discuss the effective mechanism of vitiligo treatment by compound glycyrrhizin combined with fractional laser and triamcinolone acetonide injection.

Methods: Forty-two patients with vitiligo vulgaris in the stable phase were classified into combined group (19 cases) and medicine group (23) admitted in dermatology department, Baoding First Central Hospital from January 2017 to July 2018. Both groups took 50mg compound glycyrrhizin orally three times per day, and applied halometasone cream externally once per day. Based on this treatment method, after the combined group adopted fractional laser, triamcinolone acetonide injection encapsulation was used immediately. After the treatment for six months, the curative effect was judged for both groups. Flow cytometry was used to test the changes of $\mathrm{T}$ lymphocyte subpopulation in peripheral blood before and after treatment. Meanwhile, immunohistochemical method was adopted to determine $\mathrm{CD}_{4}^{+}$and $\mathrm{CD}_{8}^{+} \mathrm{T}$ lymphocyte expression level. Besides, the normal control group was set up.

Results: The efficacy of combined group and medicine group were $73.68 \%$ and $56.52 \%$ respectively, $P<0.05$. The comparison of $\mathrm{CD}_{3}{ }^{+}, \mathrm{CD}_{4}^{+}, \mathrm{CD}_{8}{ }^{+}$and $\mathrm{CD}_{4}{ }^{+} / \mathrm{CD}_{8}{ }^{+} \mathrm{T}$ lymphocyte level in serum and skin damage before and after treatment had no statistical significance $(P>0.05)$. Serum $\mathrm{CD}_{4}{ }^{+} \mathrm{T}$ cells of vitiligo patients reduced, compared with the normal control group $(P<0.05)$, and $\mathrm{CD}_{4}{ }^{+} / \mathrm{CD}_{8}{ }^{+}$declined $(P<0.05) . \mathrm{CD}_{4}{ }^{+}$and $\mathrm{CD}_{8}{ }^{+}$ T lymphocytes at the skin damage of patients increased, compared with normal control group $(P<0.05)$. Conclusions: Compound glycyrrhizin combined with fractional laser and triamcinolone acetonide injection has good clinical effect in the treatment of vitiligo vulgaris in the stable phase, and its effective mechanism may have nothing to do with $\mathrm{T}$ lymphocyte subpopulation.
\end{abstract}

KEYWORDS: Vitiligo; Compound glycyrrhizin; Fractional laser; T lymphocyte subpopulation.

How to cite this:

doi: https://doi.org/10.12669/pjms.38.1.4412

Li L, Gao L, Zhao Y. Effect of vitiligo treatment by compound Glycyrrhizin combined with fractional laser and Triamcinolone Acetonide injection on T Lymphocyte subpopulation. Pak J Med Sci. 2022;38(1):201-206. doi: https://doi.org/10.12669/pjms.38.1.4412

This is an Open Access article distributed under the terms of the Creative Commons Attribution License (http://creativecommons.org/licenses/by/3.0), which permits unrestricted use, distribution, and reproduction in any medium, provided the original work is properly cited.

1. Ling Li,

2. Lei Gao,

3. Yifan Zhao,

1,3: Department of Dermatology,

Baoding First Central Hospital, Baoding, Hebei 071000, China.

2. Department of Dermatology, Baoding No.1 Hospital, Baoding, Hebei 071000, China.

Correspondence:

Ling Li,

Department of Dermatology,

Baoding First Central Hospital,

Baoding, Hebei 071000, China.

E-mail: hbnjvr@sina.com

* Received for Publication:

* Revision Received:

* Revision Accepted: *
March 12, 2021

July 12, 2021

July 25,2021

\section{INTRODUCTION}

Vitiligo is a primary local or extensive skin mucosa depigmentation skin disease. The average morbidity in the world is $0.5-1.0 \%,{ }^{1}$ with racial difference. The darker the skin color, the higher the morbidity. Generally, adolescents show a high morbidity. ${ }^{2}$ Since this disease affects appearance, it often brings about psychological pressure to patients and affects their normal social life. ${ }^{3-4}$ The pathogenesis of vitiligo is still unclear, and there is no specific therapy. We applied compound glycyrrhizin combined with fractional laser and 
triamcinolone acetonide injection to treat vitiligo, and gained the good effect. We used compound glycyrrhizin combined with dot-matrix laser and triamcinolone acetonide injection to treat vitiligo and achieved good results, and discussed its effective mechanism, which is reported as follows.

\section{METHODS}

Forty-two patients suffering from vitiligo vulgaris in the stable phase were included in the study which was conducted in the dermatology department, Baoding First Central Hospital were from January 2017 to July 2018.

Ethical approval: The study was approved by the Institutional Ethics Committee of Baoding First Central Hospital (No.: [2019]-001; date: 11 June, 2019), and written informed consent was obtained from all participants.

\section{Inclusion criteria:}

1. Conformity to diagnosis standard of vitiligo vulgaris, no development of rash within six months;

2. Zood compliance, willing to receive examination and subsequent visit at a regular interval;

3. No systematic or local use of immunosuppressor within three months.

\section{Exclusion criteria:}

1. Patients with severe hepatic and renal function incompetence, hypertension, diabetes and heart disease;

2. Women in gravidity and lactation period;

3. Patients with skin cancer and photaesthesia. The patients selected were classified into medicine group (23 cases) and combined group at random (19 cases).

Compound glycyrrhizin was applied for the medicine group, while compound glycyrrhizin combined with $\mathrm{CO}_{2}$ fractional laser and triamcinolone acetonide injection was applied for the combined group. There were 13 male patients and 10 female patients in the medicine group, with the age of $3 \sim 68$ and average age of $35.14 \pm 15.5$. The course of disease was 1 360 months, and the average course of disease was $(72.95 \pm 76.34)$ months. There were 10 male patients and nine female patients in the combined group, with the age of $6 \sim 55$ and average age of $34.05 \pm 12.45$. The course of disease was 2 360 months, and the average course of disease was (76.26 \pm 67.23$)$ months. Clinical data comparison of both groups in gender, age and course of disease had no statistical significance $(p>0.05)$. Pathologic biopsy was completed for 10 patients in the medicine group before and after treatment, while hat was completed for 11 patients in the combined group. 11 patients with normal skin damage in the control group were chosen. The comparison of patients and the control group in gender, age and part had no statistical significance $(p>0.05)$.

Both groups took $50 \mathrm{mg}$ compound glycyrrhizin tablet orally three times per day (trade name: Meineng, produced by Minophagen Pharmaceutical Co., Ltd. and sold by Shenzhen Jian'an Medical Company), and proper amount of halometasone cream (trade name: Aoneng, produced by Hong Kong Bright Future Pharmaceutical Co., Ltd.) was used externally once per day. On this basis, the combined groupwas treated with $\mathrm{CO}_{2}$ fractional laser (produced by Jilin King Laser Technology Co., Ltd.). The energy density was $20 \sim 40 \mathrm{~mJ} /$ pulse, and the coverage was 10 20\%. After treatment, triamcinolone acetonide injection (trade name: Transton, produced by Kuning Jida Pharmaceutical Co., Ltd.) was encapsulated for ten minutes immediately once per three weeks. The curative effect was observed after 6 months. After the course of treatment ended, follow-up visit continued, and adverse effects and recurrence rate were evaluated. Observation Indicators: (1) Clinical effect criteria ${ }^{5}$ : healed: vitiligo fades away completely; significantly effective: vitiligo area decreases or fades away, and the area of normal skin color recovered is $50 \%$ or above; improved: vitiligo fades away or decreases; ineffective: no pigment of vitiligo regenerates or the scope expands. Effective rate $=$ cure rate + significantly effective rate. (2) Adverse effects: adverse effects of both groups were observed. (3) Peripheral blood $\mathrm{T}$ lymphocyte subpopulation expression: $2 \mathrm{ml}$ peripheral fasting venous blood was gathered for both groups, and flow cytometry (produced by American Beckmann Corporation, model: Epics XL) was used to detect $\mathrm{CD}_{3}{ }^{+}, \mathrm{CD}_{4}^{+}$, $\mathrm{CD}_{8}^{+}$and $\mathrm{CD}_{4}^{+} / \mathrm{CD}_{8}^{+} \mathrm{T}$ lymphocyte level. (4) $\mathrm{CD}_{4}$ and $\mathrm{CD}_{8} \mathrm{~T}$ lymphocyte immunohistochemistry was conducted for skin damage tissues.

Statistical Analysis: SPSS13.0 statistics software was used for analysis. Measurement data were expressed with $\bar{x} \pm \mathrm{s}$, and independent $\mathrm{t}$ test was used for intra-group comparison. Enumeration data were expressed with frequency and percentage, and $\chi^{2}$ test was adopted for intergroup comparison. $\mathrm{P}<0.05$ means differences have statistical significance.

\section{RESULTS}

Three patients cured; eleven had significant effect; four improved; one had no effect; the 


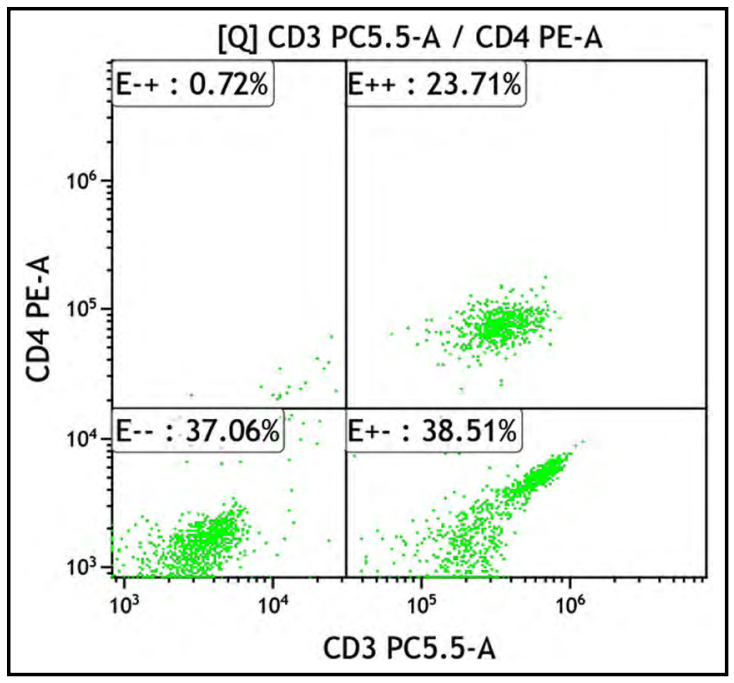

Fig.1: $\mathrm{CD}_{4}^{+} \mathrm{T}$ lymphocyte expression of combined group before treatment.

effective rate was $73.68 \%$. In the medicine group, two patients cured; eleven had significant effect; seven improved; three had no effect; the effective rate was $56.52 \%$. The effect rate of combined group was obviously higher than that of medicine group $(P<0.05)$.

Three patients felt causalgia, and was relieved after the treatment ended, without special treatment; two showed facial edema, was relieved spontaneously after drug withdrawal and quitted the experiment. (Fig.1-3) Comparison of $\mathrm{T}$ lymphocyte subpopulation expression level of both groups before and after treatment is shown in Table-I. After treatment, $\mathrm{CD}_{3}^{+}$and $\mathrm{CD}_{4}^{+} \mathrm{T}$ lymphocyte of both groups slightly increased; $\mathrm{CD}_{8}^{+}$ T lymphocyte slightly declined; $\mathrm{CD}_{4}^{+} / \mathrm{CD}_{8}^{+}$did not rise significantly; the comparison before and after treatment had no statistical significance $(p>0.05)$. $\mathrm{T}$ lymphocyte subpopulation of both groups had

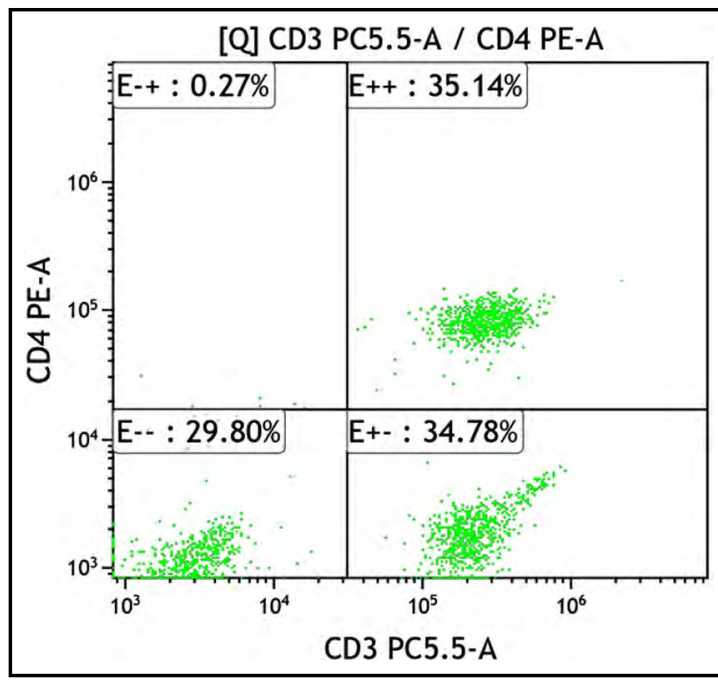

Fig.2: $\mathrm{CD}_{4}^{+} \mathrm{T}$ lymphocyte expression of combined group after treatment.

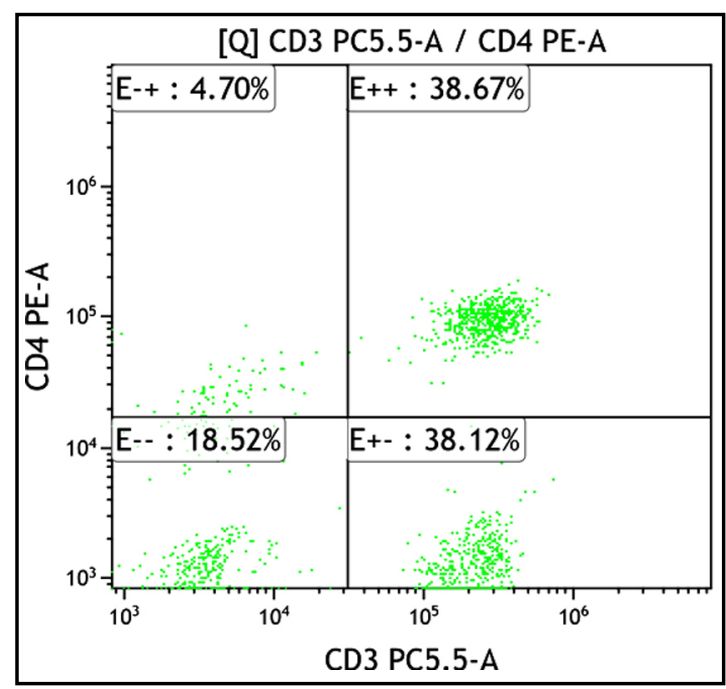

Fig.3: $\mathrm{CD}_{4}^{+} \mathrm{T}$ lymphocyte expression of normal control group.

Table-I: Comparison of T lymphocyte subpopulation expression level of combined group and medicine group before and after treatment $(\bar{x} \pm \mathrm{s})$.

\begin{tabular}{lccccc}
\hline Group & $n$ & $\mathrm{CD}_{3}^{+}(\%)$ & $\mathrm{CD}_{4}^{+}(\%)$ & $\mathrm{CD}_{8}^{+}(\%)$ & $\mathrm{CD}_{4}^{+} / \mathrm{CD}_{8}^{+}$ \\
\hline Combined group & 19 & & & & \\
Before treatment & & $53.64 \pm 9.84$ & $32.02 \pm 7.98$ & $22.85 \pm 4.10$ & $1.41 \pm 0.29$ \\
Treatment for 6 months & & $55.96 \pm 8.97$ & $33.12 \pm 7.39$ & $21.94 \pm 2.64$ & $1.51 \pm 0.27$ \\
p & & 0.452 & 0.662 & 0.422 & 0.278 \\
Medicine group & 23 & & & & \\
Before treatment & & $52.79 \pm 9.82$ & $31.64 \pm 8.02$ & $20.80 \pm 3.19$ & $1.54 \pm 0.43$ \\
Treatment for 6 months & & $53.68 \pm 9.53$ & $34.77 \pm 8.71$ & $20.63 \pm 2.51$ & $1.70 \pm 0.42$ \\
p & & 0.755 & 0.211 & 0.841 & 0.218 \\
\hline
\end{tabular}

Note: Comparison of both groups before and after treatment, $\mathrm{P}>0.05$. 
Ling Li et al.

Table-II: Comparison of T lymphocyte subpopulation expression level of vitiligo patients and normal control group $(\bar{x} \pm \mathrm{s})$.

\begin{tabular}{lccccc}
\hline Item & $n$ & $C D_{3}^{+}(\%)$ & $C D_{4}^{+}(\%)$ & $C D_{8}^{+}(\%)$ & $\mathrm{CD}_{4}^{+} / \mathrm{CD}_{8}^{+}$ \\
\hline Vitiligo patients & 42 & $53.17 \pm 9.72$ & $31.81 \pm 7.91$ & $21.72 \pm 3.73$ & $1.48 \pm 0.38$ \\
Control group & 23 & $57.33 \pm 10.11$ & $36.90 \pm 9.22$ & $20.43 \pm 2.76$ & $1.82 \pm 0.47$ \\
$\mathrm{p}$ & & 0.115 & 0.031 & 0.117 & 0.004 \\
\hline
\end{tabular}

Note: $\mathrm{CD}_{4}^{+}$and $\mathrm{CD}_{4}^{+} / \mathrm{CD}_{8}^{+}$Comparison between vitiligo patients and normal control group, $\mathrm{P}<0.05$.

Table-III: $\mathrm{CD}_{4}{ }^{+}$and $\mathrm{CD}_{8}{ }^{+} \mathrm{T}$ lymphocyte expression level of combined group and medicine group in the skin damage part before and after treatment $(\bar{x} \pm \mathrm{s})$.

\begin{tabular}{lcccc}
\hline Group & $\mathbf{n}$ & $C D_{4}^{+}$ & $C D_{8}^{+}$ & $C D_{4}^{+} / C D_{8}^{+}$ \\
\hline Combined group & 11 & & & \\
Before treatment & & $11.97 \pm 6.09$ & $8.75 \pm 4.84$ & $1.57 \pm 0.61$ \\
Treatment for 6 months & & $10.03 \pm 3.99$ & $8.27 \pm 4.52$ & $1.46 \pm 0.56$ \\
p & & 0.131 & 0.676 & 0.452 \\
Medicine group & 10 & & & \\
Before treatment & & $11.57 \pm 4.85$ & $9.67 \pm 4.73$ & $1.59 \pm 0.67$ \\
Treatment for 6 months & & $9.27 \pm 5.18$ & $8.70 \pm 4.56$ & $1.41 \pm 0.90$ \\
p & & 0.081 & 0.423 & 0.394 \\
\hline
\end{tabular}

Note: comparison of two vitiligo groups before and after treatment, $\mathrm{P}>0.05$.

no obvious change before and after treatment. The comparison between two groups of vitiligo patients before treatment and normal control group is shown in Table-II. Both $\mathrm{CD}_{4}^{+} \mathrm{T}$ lymphocyte and $\mathrm{CD}_{4}^{+} / \mathrm{CD}_{8}^{+}$of vitiligo patients declined, compared with the normal control group $(P<0.05)$. The comparison of $\mathrm{CD}_{3}{ }^{+}$and $\mathrm{CD}_{8}^{+} \mathrm{T}$ lymphocyte had no statistical significance $(P>0.05)$.

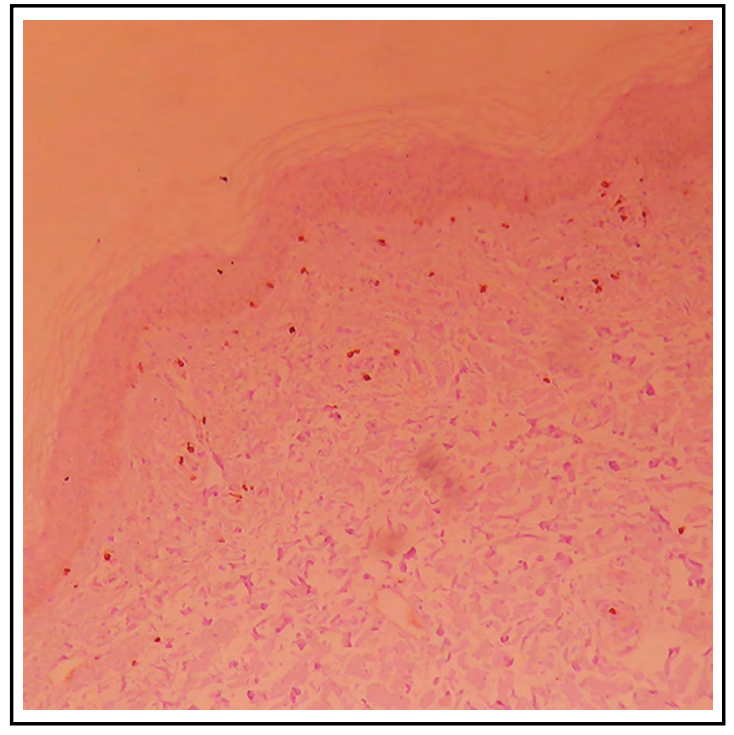

Fig.4: $\mathrm{CD}_{4}^{+}$lymphocyte expression in skin damage tissue of normal control group $(\times 100)$.
T lymphocyte subpopulation expression level in damaged skin (Fig.4-6) The manifestation of positive $\mathrm{CD}_{4}$ and $\mathrm{CD}_{8} \mathrm{~T}$ lymphocyte is brown yellow cytomembrane. After treatment, $\mathrm{CD}_{4}^{+}$and $\mathrm{CD}_{8}^{+} \mathrm{T}$ lymphocytes of both groups slightly declined, and $\mathrm{CD}_{4}^{+} / \mathrm{CD}_{8}^{+}$slightly decreased. The comparison of both groups before and after treatment had no statistical significance ( $p>0.05$, shown in Table-III).

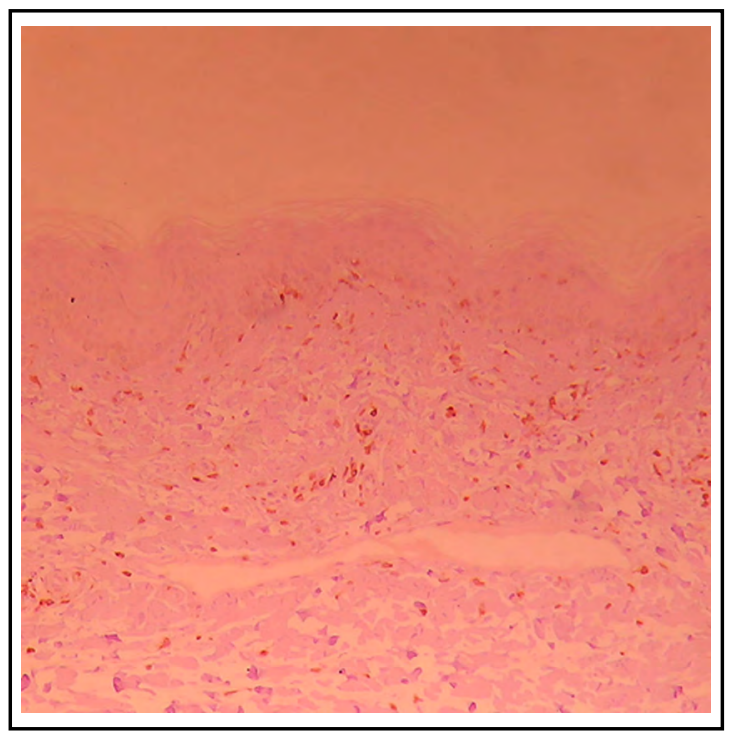

Fig.5: $\mathrm{CD}_{4}^{+} \mathrm{T}$ lymphocyte expression in skin damage tissue before combined treatment $(\times 100)$. 


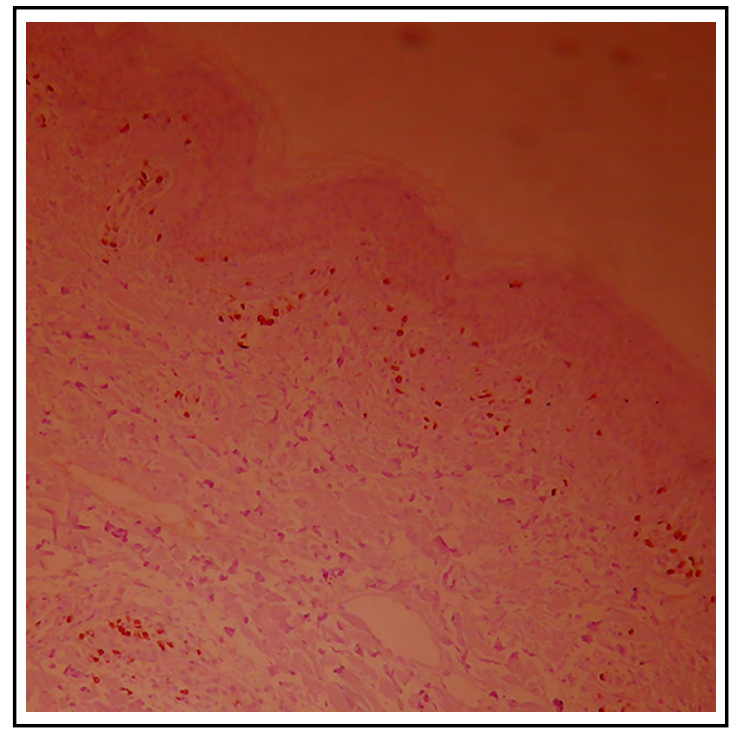

Fig.6: $\mathrm{CD}_{4}^{+} \mathrm{T}$ lymphocyte expression in skin damage tissue after combined treatment $(\times 100)$.

$\mathrm{CD}_{4}^{+}$and $\mathrm{CD}_{8}^{+} \mathrm{T}$ lymphocyte expression level of vitiligo patients increased, compared with normal control group $(p<0.05)$, and $\mathrm{CD}_{8}^{+} \mathrm{T}$ lymphocyte expression level of vitiligo patients increased, compared with normal control group $(p<0.05$, shown in Table-IV).

\section{DISCUSSION}

In recent years, comprehensive treatment of vitiligo has been recognized by people from all walks of life. The treatment method of oral administration and external use of drugs combined with phototherapy is often regarded as the clinical treatment method. The main compositions of compound glycyrrhizin include glycyrrhizic acid extracted from liquorice, glycine and cysteine which have the effect of anti-inflammation, anti-allergic reaction and immunoregulation as well as the effect of parahormone. ${ }^{6-8}$ Compound glycyrrhizin can promote immunoregulation, regulate $\mathrm{T}$ cell activation, induce interferon generation, activate NK cells, enhance T lymphocyte differentiation capacity, reduce melanocyte damage, recover melanocyte function, and recover normal skin color..$^{9,10}$

Fractional laser can gasify and peel epidermis and dermis, with small slight thermal injury area. Besides, it can promote skin healing, greatly relieve patients' pains and shorten treatment time. ${ }^{11,12}$ Some researchers have found that, fractional laser combined with multiple therapies can achieve the better effect on persistent vitiligo treatment. ${ }^{13-15}$ The principle of vitiligo treatment by fractional laser is as follows: it stimulates the skin damage area to secrete the inflammatory cytokine so as to promote melanocyte proliferation and migration, construct drug delivery path and facilitate external drug absorption. Fractional laser can form microscopic pores consistent with the light beam in the therapy area. The fine pore diameter is beneficial to drug delivery. After fractional laser treatment, triamcinolone acetonide injection is encapsulated immediately, which can significantly improve the curative effect and stimulate percutaneous immune induction

$\mathrm{T}$ lymphocyte as a major regulating cell and effector cell can recognize and dispose antigen, generate multiple cytokines, maintain steady state of system and balance immunity. The laboratory investigation showed that, after $\mathrm{CD} 4^{+}$ $\mathrm{T}$ lymphocytes in the mice were removed, $\mathrm{CD}^{+}$ $\mathrm{T}$ lymphocytes increased, and more than a half of mice showed the features of vitiligo. ${ }^{16,17}$ The Chinese investigator Jiang $\mathrm{Yu}$ found that the immunity of patients with generalized vitiligo was low, $\mathrm{CD}^{+} \mathrm{T}$ lymphocyte declined, and $\mathrm{CD} 8^{+}$ $\mathrm{T}$ lymphocyte rose. After the immunomodulatory was used, $\mathrm{CD}^{+} \mathrm{T}$ lymphocyte rose, and $\mathrm{CD} 8^{+} \mathrm{T}$ lymphocyte declined to different degree. Besides, patients' immunity improved. ${ }^{18,19}$

Limitations of the study: The sample size should be expanded to further study whether the effective therapeutic mechanism of compound glycyrrhizin combined with fractional laser and triamcinolone acetonide injection in vitiligo treatment is related to $\mathrm{T}$ lymphocyte subpopulation regulation.

Table-IV: $\mathrm{CD}_{4}^{+}$and $\mathrm{CD}_{8}^{+} \mathrm{T}$ lymphocyte expression level in the skin damage part of vitiligo patients and normal control group $(\bar{x} \pm \mathrm{s})$.

\begin{tabular}{lcccc}
\hline Item & $n$ & $C D_{4}^{+}$ & $C D_{8}^{+}$ & $C D_{4}^{+} / C D_{8}^{+}$ \\
\hline Vitiligo patients & 21 & $11.78 \pm 5.49$ & $8.73 \pm 4.67$ & $1.58 \pm 0.64$ \\
Control group & 11 & $8.30 \pm 4.98$ & $5.49 \pm 3.33$ & $2.00 \pm 0.65$ \\
$\mathrm{p}$ & & 0.003 & 0.001 & 0.003 \\
\hline
\end{tabular}

Note: comparison of $\mathrm{CD}^{+}, \mathrm{CD}^{+}$and $\mathrm{CD}^{+} / \mathrm{CD}^{+}$between vitiligo patients and normal control group, $\mathrm{P}<0.05$ 
The reports on the detection of peripheral blood $\mathrm{T}$ lymphocyte subpopulation of vitiligo patients are different, and most studies hold that $\mathrm{CD}^{+} \mathrm{T}$ lymphocyte declines, $\mathrm{CD} 8^{+} \mathrm{T}$ lymphocyte rises and $\mathrm{CD}^{+} / \mathrm{CD}^{+}$lowers. This study found that, $\mathrm{CD}^{+} \mathrm{T}$ lymphocyte of vitiligo patients declined; $\mathrm{CD}^{+} / \mathrm{CD}^{+}$lowered, compared with the normal control group; $\mathrm{CD} 8^{+} \mathrm{T}$ lymphocyte rise was not found. This might be related to vitiligo vulgaris in the stable phase. Some studies believed that, $\mathrm{T}$ lymphocyte subpopulation of vitiligo patients in the progressive phase changes more obviously than normal people. ${ }^{20}$ In the damaged skin, $\mathrm{CD} 4{ }^{+} \mathrm{T}$ and $\mathrm{CD}^{+} \mathrm{T}$ lymphocytes of vitiligo patients rose, compared with the normal control group, and $\mathrm{T}$ lymphocyte migration might exist in blood and damaged skin of vitiligo patients.

\section{CONCLUSIONS}

Compound glycyrrhizin combined with fractional laser and triamcinolone acetonide injection in vitiligo treatment showed the definite curative effect. After treatment, T lymphocyte subpopulation changes in serum and damaged skin had no statistical significance. Thus, it is inferred that the effective mechanism of combined and independent treatment of vitiligo in the stable phase may have no obvious relation with $\mathrm{T}$ lymphocyte subpopulation.

Source of funding: It was sponsored by Hebei Provincial Administration of Traditional Chinese Medicine (HY 2019 CX); sHebei Provincial Administration of Traditional Chinese Medicine (No.: 2019366).

\section{Conflicts of interest: None.}

\section{REFERENCES}

1. Ezzedine K, Lim HW, Suzuki T, Katayama I, Hamzavi I, Lan $\mathrm{CC}$, et al. Revised classification/nomenclature of vitiligo and related issues: the Vitiligo Global Issues Consensus Conference. Pigment Cell Melanoma Res. 2012;25(3):E1-E13. doi: 10.1111/j.1755-148X.2012.00997.x

2. Majumder PP, Nordlund JJ, Nath SK. Pattern of familial aggregation of vitiligo. Arch Dermatol. 1993;129(8):994-998.

3. Jimbow K: Vitiligo Therapeutic advances. J Dermatol Clm. 1998;16(2):399-407.

4. Mou KH, Han D, Liu WL, Li P. Combination therapy of orally administered glycyrrhizin and UVB improved active-stage generalized vitiligo. Brazilian J Med Biolog Res. 2016;49(8) doi: 10.1590/1414-431X20165354

5. Yu JJ, Zhang CS, Coyle ME, Du Y, Zhang AL, Guo X, et al Compound glycyrrhizin plus conventional therapy for psoriasis vulgaris: a systematic review and meta-analysis of randomized controlled trials. Curr Med Res Opin. 2016;33(2):279-287. doi: 10.1080/03007995.2016.1254605
6. Omi $\mathrm{T}$, Numano $\mathrm{K}$. The role of the $\mathrm{CO} 2$ laser and fractional $\mathrm{CO} 2$ laser in dermatology. Laser Ther. 2014;23(1):49-60. doi: 10.5978/ islsm.14-RE-01

7. Li L, Wu Y, Li L, Sun Y, Qiu L, Gao XH, et al. Triple combination treatment with fractional $\mathrm{CO} 2$ laser plus topical betamethasone solution and narrowband ultraviolet $\mathrm{B}$ for refractory vitiligo: a prospective, randomized half-body, comparative study. Dermatol Ther. 2015,28(3):131-134. doi: 10.1111/dth.12202

8. Peng FJ, Zhang TX, Yuan MR.Influence of compound glycyrrhizin on liver functions, liver fibrosis indexes and inflammatory factors of patients with chronic hepatitis B. J Hainan Med Uni. 2017;23(4).

9. Rachmut IH, Samuels N, Melnick SJ, Ramachandran C, Sharabi Y, Pavlovsky A, et al. Immunomodulatory effects of the botanical compound LCS101: implications for cancer treatment. Oncotargets Ther. 2013;6(6):437-445. doi: 10.2147/OTT.S42038

10. Yue ZF, Han XS, Dong Y, Tian K, Guo X, Liu XD.Effect of compound glycyrrhizin combined with NB-UVB on $T$ lymphocyte subsets and related cytokines in patients with psoriasis vulgaris. J Hainan Med Uni. 2016;22(18).

11. Zhao JH, Richer V, Al JM, Zandi S, Kollias N, Kalia S, et al.Fluorescence excitation-emission matrix spectroscopy of vitiligo skin in vivo (Conference Presentation).Photonic Therapeutics and Diagnostics XII. Int Soc Optics Photonics. 2016:968911. doi: $10.1117 / 12.2214210$

12. Li YL, Qi RQ, Yang Y, Wang HX, Jiang HH, Li ZX, et al. Screening and identification of differentially expressed serum proteins in patients with vitiligo using two-dimensional gel electrophoresis coupled with mass spectrometry. Molecular Medicine Reports, 2018;17(2):2651. doi:10.3892/mmr.2017.8159.

13. Padma D, Bhat KMR. Effect of Extracellular Matrix Produced by the Fibroblasts Derived from the Vitiligo Skin on Survival and Function of Melanocytes. Adv Sci Letters. 2017;23(3):1904-1909. doi: 10.1166/asl.2017.8526

14. Blokzijl A, Chen LE, Gustafsdottir SM, Vuu J, Ullenhag G, Kampe O, et al. Elevated Levels of SOX10 in Serum from Vitiligo and Melanoma Patients, Analyzed by Proximity Ligation Assay. Plos One. 2016;11(4):e0154214. doi: 10.1371/journal. pone. 0154214

15. Yang Y, Zapata L, Rodgers C, Hernandez K, Iyer M, Jia G, et al. Quality of life in patients with vitiligo using the short form 36 . Brit J Dermatol. 2017;177. doi: 10.1111/bjd.15936

16. Jacquemin C, Taieb A, Boniface K, Seneschal J. 088 Imbalance of peripheral $\mathrm{T}$ follicular helper lymphocyte subsets in active vitiligo. J Investigative Dermatol. 2018;138(5):S15. doi: 10.1016/j. jid.2018.03.092

17. Kaur M, Bagga PK, Kaur T, Kataria AS. Evaluation of Histologically and Histochemically Proven Cases of Vitiligo and its Correlation with CD4+ and CD8+ Lymphocyte Counts using Flow Cytometry. J Clin Diagn Res. 2017;11(5):EC09. doi: 10.7860/JCDR/2017/25665.9821

18. Xie H, Zhou F, Liu L, Zhu G, Li O, Li C, et al. Vitiligo: How do oxidative stress-induced autoantigens trigger autoimmunity? J Dermatolog Sci. 2016;81(1):3-9. doi: 10.1016/j. jdermsci.2015.09.003

19. Solak B, Dikicier BS, Cosansu NC, Erdem T. Neutrophil to lymphocyte ratio in patients with vitiligo. Adv Dermatol Allergology/postepy Dermatologii I Alergologii. 2017;34(5):468470. doi: 10.5114 /ada.2017.71114

20. Speeckaert R, Van G N. Targeting CTLA-4, PD-L1 and IDO to modulate immune responses in vitiligo. Experimen Dermatol. 2016;26(7). doi: 10.1111/exd.13069

Authors' Contributions: LL: Designed this study and prepared this manuscript, and is responsible and accountable for the accuracy or integrity of the work. LG \& YZ: Collected and analyzed clinical data. Note: Ling Li and Lei Gao contribute to this work equally. 